\title{
Vacuum-Stimulated Raman Scattering Based on Adiabatic Passage in a High-Finesse Optical Cavity
}

\author{
M. Hennrich, T. Legero, A. Kuhn, and G. Rempe \\ Max-Planck-Institut für Quantenoptik, Hans-Kopfermann-Strasse 1, D-85748 Garching, Germany
}

(Received 21 August 2000)

\begin{abstract}
We report on the first observation of stimulated Raman scattering from a $\Lambda$-type three-level atom, where the stimulation is realized by the vacuum field of a high-finesse optical cavity. The scheme produces one intracavity photon by means of an adiabatic passage technique based on a counterintuitive interaction sequence between pump laser and cavity field. This photon leaves the cavity through the less-reflecting mirror. The emission rate shows a characteristic dependence on the cavity and pump detuning, and the observed spectra have a subnatural linewidth. The results are in excellent agreement with numerical simulations.
\end{abstract}

PACS numbers: 32.80.Qk, 03.67.-a, 42.50.Ct, 42.65.Dr

In the past few years, interesting proposals on the generation of nonclassical states of light in optical cavities $[1,2]$ and on the controlled generation of single photons from such cavities $[3,4]$ were made. All of these schemes are based on a technique known as STIRAP (stimulated Raman scattering involving adiabatic passage) [5,6] or a variant thereof, and incorporate the time dependent interaction of an atom with the field mode of an optical cavity. The operation principle is related to that of a Raman laser [7], with the difference that now a single atom interacts with an empty cavity mode. Other schemes for the preparation of Fock states are based on vacuum Rabi oscillations or, more generally, $\pi$ pulses in a two-level atom. In these cases, the need for a long-lived excited atomic state restricts experiments to the microwave regime $[8,9]$, where the photon remains stored in a high- $Q$ cavity.

Here, we report on the experimental realization of an excitation scheme that allows one to emit a visible photon into a well-defined mode of an empty cavity. This photon then leaves the cavity in a known direction. Our method is based on the single-photon generation scheme discussed in [4]. It relies on STIRAP [5,6], but, instead of using two delayed laser pulses, we have only one exciting pump laser, combined with a strong coupling of a single atom to a single cavity mode $[10,11]$. This strong coupling induces the anti-Stokes transition of the Raman process.

Figure 1 depicts the excitation scheme for the ${ }^{85} \mathrm{Rb}$-atoms used in our experiment. A $\Lambda$-type three-level scheme is realized by the two $5 S_{1 / 2}$ hyperfine ground states $F=3$ and $F=2$, which we label $|u\rangle$ and $|g\rangle$, respectively. The $F=3$ hyperfine level of the electronically excited state, $5 P_{3 / 2}$, forms the intermediate state, $|e\rangle$. The atom interacts with a single-mode of an optical cavity, with states $|0\rangle$ and $|1\rangle$ denoting zero and one photon in the mode, respectively. The cavity resonance frequency, $\omega_{C}$, is close to the atomic transition frequency between states $|e\rangle$ and $|g\rangle$, but far off resonance from the $|e\rangle$ to $|u\rangle$ transition. Hence, only the product states $|e, 0\rangle$ and $|g, 1\rangle$ are coupled by the cavity. For this transition, the vacuum
Rabi frequency,

$$
2 g(t)=2 g_{0} \exp \left[-\left(\frac{t v}{w_{C}}\right)^{2}\right],
$$

is time dependent since the atom moves with velocity $v$ across the waist $w_{C}$ of the Gaussian cavity mode. Its peak amplitude is given by the atom-cavity coupling coefficient at an antinode, $g_{0}$.

In addition to the interaction with the cavity mode, the atom is exposed to a pump laser beam which crosses the cavity axis at right angle. This beam is placed slightly downstream in the path of the atoms (by $\delta_{x}$ with respect to the cavity axis) and has a waist $w_{P}$, therefore causing a time dependent Rabi frequency

$$
\Omega_{P}(t)=\Omega_{0} \exp \left[-\left(\frac{t v-\delta_{x}}{w_{P}}\right)^{2}\right] .
$$

The pump frequency is near resonant with the transition between $|u, 0\rangle$ and $|e, 0\rangle$, thereby coupling these states.

In a frame rotating with the cavity frequency and the pump laser frequency, the Hamiltonian is given by

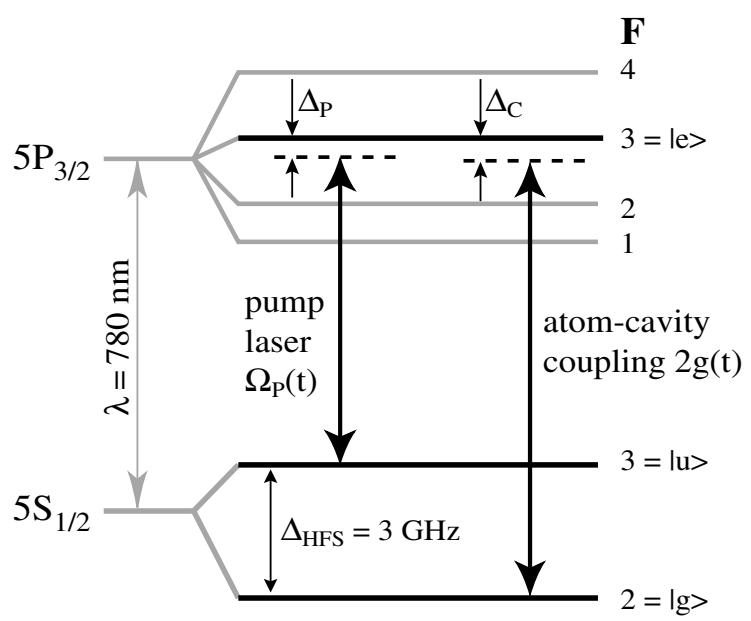

FIG. 1. Scheme of the relevant levels, transitions, and detunings of the ${ }^{85} \mathrm{Rb}$ atom coupled to the pump laser and the cavity. 


$$
\begin{aligned}
H(t)= & \hbar\left[\Delta_{P}|u\rangle\left\langle u\left|+\Delta_{C}\right| g\right\rangle\langle g|\right. \\
& +g(t)\left(|e\rangle\left\langle g\left|a+a^{\dagger}\right| g\right\rangle\langle e|\right) \\
& \left.+\frac{1}{2} \Omega_{P}(t)(|e\rangle\langle u|+| u\rangle\langle e|)\right] .
\end{aligned}
$$

Here, $\Delta_{C}$ and $\Delta_{P}$ denote the detunings of the cavity and the pump beam from their respective atomic resonances, and $a$ and $a^{\dagger}$ are the annihilation and creation operators of the cavity field. The pump beam is treated semiclassically. On Raman resonance, i.e., for $\Delta_{C}=\Delta_{P}$, one of the eigenstates of this interaction Hamiltonian reads

$$
\left|a^{0}(t)\right\rangle=\frac{2 g(t)|u, 0\rangle-\Omega_{P}(t)|g, 1\rangle}{\sqrt{4 g^{2}(t)+\Omega_{P}^{2}(t)}} .
$$

This is a dark state without any contribution from the electronically excited level $|e, 0\rangle$. Therefore losses due to spontaneous emission cannot occur, provided the state vector of the system, $|\Psi\rangle$, follows $\left|a^{0}\right\rangle$ throughout the Raman excitation.

The atom is prepared in state $|u\rangle$ before it enters the empty cavity, i.e., atom and field start in state $|u, 0\rangle$. Since the pump beam is displaced by $\delta_{x}$ with respect to the cavity axis, the atom is subject to a counterintuitive delayed pulse sequence, i.e., due to the initial condition $2 g \gg \Omega_{P}$, the evolution starts with $\left\langle\Psi \mid a^{0}\right\rangle=1$. The subsequent interaction with the pump beam leads to $\Omega_{P} \gg 2 g$, which implies the evolution of $\left|a^{0}\right\rangle$ into state $|g, 1\rangle$. Provided the state vector $|\Psi\rangle$ is able to follow, the system is transferred to $|g, 1\rangle$, and a photon is placed in the cavity mode. Since this photon is emitted with the cavity energy decay rate, $2 \kappa$, the empty cavity state, $|g, 0\rangle$, is finally reached and the atom-cavity system decouples from any further interaction.

This simple excitation scheme relies on three conditions. First, the detunings of the cavity, $\Delta_{C}$, and of the pump pulse, $\Delta_{P}$, must allow a Raman transition, i.e.,

$$
\left|\Delta_{C}-\Delta_{P}\right|<2 \kappa .
$$

Second, the condition for $|\Psi\rangle$ adiabatically following $\left|a^{0}\right\rangle$ must be met $[4,5]$,

$$
\left(2 g_{0} w_{C} / v, \Omega_{0} w_{P} / v\right) \gg 1 .
$$

Third, either the interaction time must be significantly longer than $(2 \kappa)^{-1}$ to allow the emission of the photon before it is reabsorbed by the atom due to coherent population return $[4,6]$ or, alternatively, the interaction with the pump beam must be strong when the atom leaves the cavity to avoid this reverse process.

A numerical simulation for a single atom crossing the cavity is shown in Fig. 2. To include the cavity-field decay rate, $\kappa$, and the spontaneous emission rate of the atom, $\Gamma$, we have employed the density-matrix formalism described in [4]. For the resonant situation, $\Delta_{P}=\Delta_{C}=$ 0 , shown here, the total emission probability, $P_{\text {emit }}$, is expected to reach $90 \%$. For the considered waists and amplitudes, Fig. 2(c) shows that $P_{\text {emit }}$ reaches its maximum for $\delta_{x} / v=45 \mu \mathrm{s}$. Note also that $P_{\text {emit }}$ is vanishingly small if
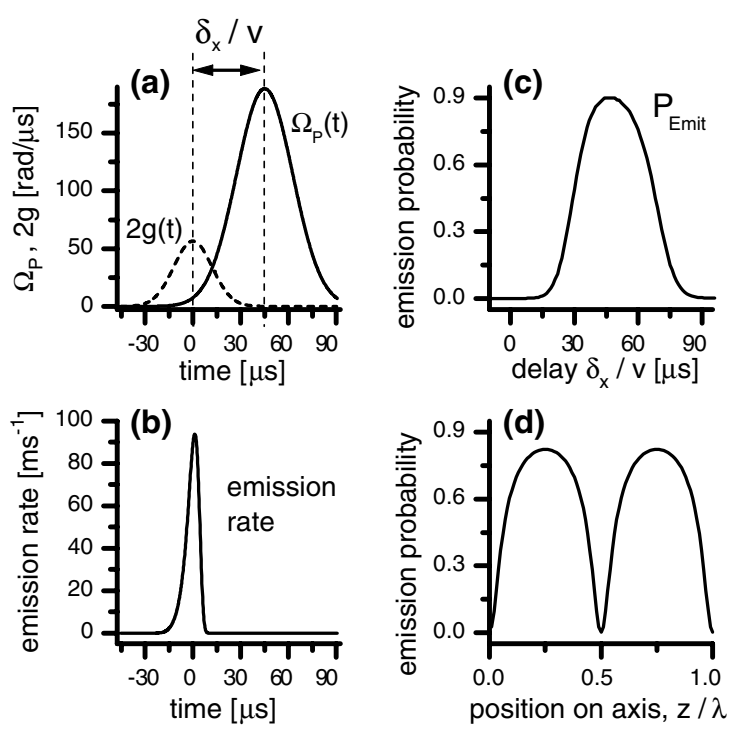

FIG. 2. Simulation of a resonant atom-cavity interaction sequence for a cavity decay constant, $2 \kappa=2 \pi \times 2.5 \mathrm{MHz}$, an atomic decay constant of $\Gamma=2 \pi \times 6 \mathrm{MHz}$, and atoms traveling at $v=2 \mathrm{~m} / \mathrm{s}$. (a) $\Omega_{P}(t)$ and $2 g(t)$ shown for experimental amplitudes and waists, $w_{C}=35 \mu \mathrm{m}$ and $w_{P}=50 \mu \mathrm{m}$. (b) Photon emission rate for a delay of $\delta_{x} / v=45 \mu \mathrm{s}$. The integral of the rate yields a total photon emission probability, $P_{\text {emit }}$, of $90 \%$. (c) $P_{\text {emit }}$ as a function of the delay, $\delta_{x} / v$, between cavity and pump interaction. (d) $P_{\text {emit }}$ as a function of the atomic position on the cavity axis for a delay of $\delta_{x} / v=35 \mu \mathrm{s}$.

the interaction with the pump beam coincides or precedes the interaction with the cavity mode. Figure 2(d) shows $P_{\text {emit }}$ as a function of the atom's position on the cavity axis for the delay realized in the experiment. Because of the standing wave mode structure, the emission probability is zero at the nodes, and shows maxima at the antinodes. Since the dependence of $P_{\text {emit }}$ on the position dependent coupling constant, $g$, is highly nonlinear and saturates for large $g$, the gaps around the nodes are much narrower than the plateaus surrounding the antinodes.

Figure 3 depicts the case where $\Delta_{P} \neq \Delta_{C}$. It is obvious that $P_{\text {emit }}$ is close to unity if the excitation is Raman resonant $\left(\Delta_{P}=\Delta_{C}\right)$. However, for the delay $\delta_{x} / v=35 \mu \mathrm{s}$ chosen here, a smaller signal is expected for $\Delta_{P}=\Delta_{C}=$ 0 , since the waist of the pump, $w_{P}$, is larger than $w_{C}$, and resonant excitation of the atom prior to the interaction with the cavity mode cannot be neglected.

To realize the proposed scheme, we have chosen the setup sketched in Fig. 4. A cloud of ${ }^{85} \mathrm{Rb}$ atoms is prepared in the $5 S_{1 / 2}, F=3$ state and released from a magnetooptical trap (MOT) at a temperature of $\approx 10 \mu \mathrm{K}$. A small fraction (up to 100 atoms) falls through a stack of apertures and enters the mode volume of an optical cavity at a speed of $2 \mathrm{~m} / \mathrm{s}$. The cavity is composed of two mirrors with a curvature of $50 \mathrm{~mm}$ and a distance of $1 \mathrm{~mm}$. The waist of the $\mathrm{TEM}_{00}$ mode is $w_{C}=35 \mu \mathrm{m}$, and in the antinodes the coupling coefficient is $g_{0}=2 \pi \times 4.5 \mathrm{MHz}$. The finesse of 61000 corresponds to a linewidth $2 \kappa=2 \pi \times$ $2.5 \mathrm{MHz}$ (FWHM), which is significantly smaller than the 


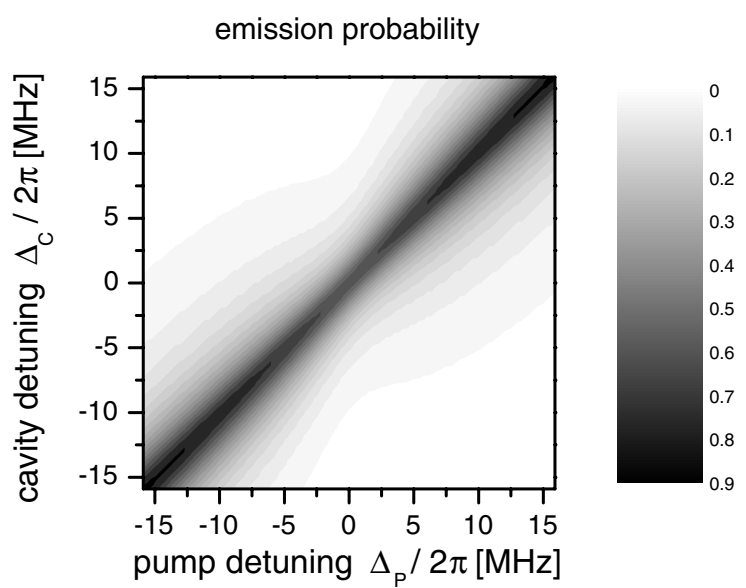

FIG. 3. Photon emission probability as a function of cavity and pump detuning, calculated for a pulse delay of $\delta_{x} / v=35 \mu \mathrm{s}$ and the parameters of Fig. 2. The chosen delay gives the best fit with the experimental data shown in Fig. 5.

natural linewidth of the ${ }^{85} \mathrm{Rb}$ atoms. While one cavity mirror is highly reflective $\left(1-R=4 \times 10^{-6}\right)$, the transmission of the other is $25 \times$ higher to emit the photons in one direction only. A single-photon counting module (SPCM) with a quantum efficiency of $50 \%$ is used to detect them.

A reference laser is used to stabilize the cavity close to resonance with the $5 S_{1 / 2}, F=2 \leftrightarrow 5 P_{3 / 2}, F=3$ transition with a lock-in technique. However, since an empty cavity is needed for the experiment, this laser is blocked $3.7 \mathrm{~ms}$ before the atoms enter the cavity.

The pump beam is close to resonance with the $5 S_{1 / 2}, F=3 \leftrightarrow 5 P_{3 / 2}, F=3$ transition and crosses the cavity transverse to its axis. This laser is focused to a waist of $50 \mu \mathrm{m}$ and has a power of $5.5 \mu \mathrm{W}$, which corresponds to a peak Rabi frequency $\Omega_{0}=2 \pi \times 30 \mathrm{MHz}$.

The desired counterintuitive pulse sequence for STIRAP is realized by time of flight. The atoms first enter the cavity mode and therefore experience a strong coupling on the anti-Stokes transition, whereas the interaction with the pump beam is delayed, since it crosses the cavity mode slightly downstream. This delay has been optimized to achieve a high flux of photons leaving the cavity.

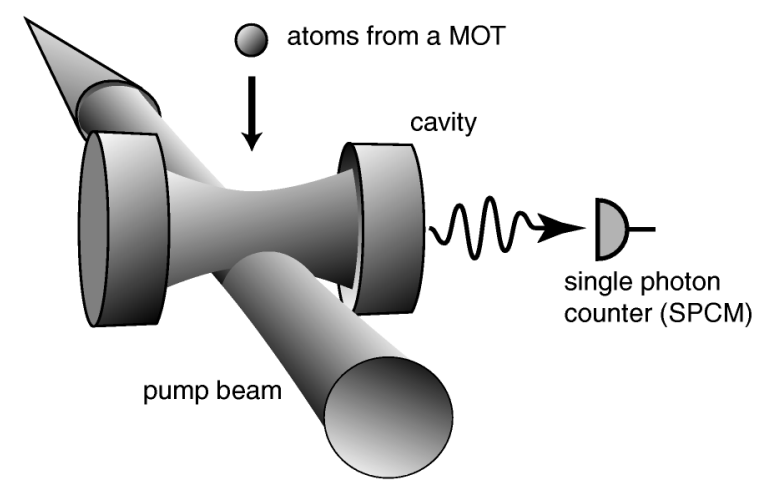

FIG. 4. Sketch of the experimental setup. The pump beam is displaced with respect to the cavity mode.
Figure 5(a) shows the number of counted photons emerging from the cavity as a function of the pump pulse detuning, $\Delta_{P}$, in case of a resonant cavity, $\Delta_{C}=0$. The detunings of the cavity and the pump laser are both adjusted by means of acousto-optic modulators. To register the data, the MOT has been loaded and dropped across the cavity 50 times. The atom cloud needs $6.5 \mathrm{~ms}$ (FWHM) to cross the cavity mode, and, within this interval, the photons emerging from the cavity are measured by the SPCM and recorded by a transient digitizer during $2.6 \mathrm{~ms}$ with a time resolution of $25 \mathrm{MHz}$. Therefore, the signal is observed for a total time of $130 \mathrm{~ms}$. Because of the dark count rate of $390 \mathrm{~Hz}$ of the SPCM, the total number of dark counts in the interval is limited to $51 \pm 7$.

In the resonant case, one expects a small probability for atomic excitation. This could lead to a small but cavity enhanced spontaneous emission into the cavity mode, as has been shown previously [12]. Our numerical simulation shows that an excited atom at the antinode emits into the resonant cavity mode with a probability that can be as high as $26 \%$, indicating that even in this case most of the spontaneously emitted photons are lost in a random direction. This loss explains the smaller peak emission rate with respect to the off-resonant cases discussed below. Note that the cavity mode covers only a small solid angle of $\approx 4 \pi \times 2.6 \times 10^{-5} \mathrm{sr}$, therefore

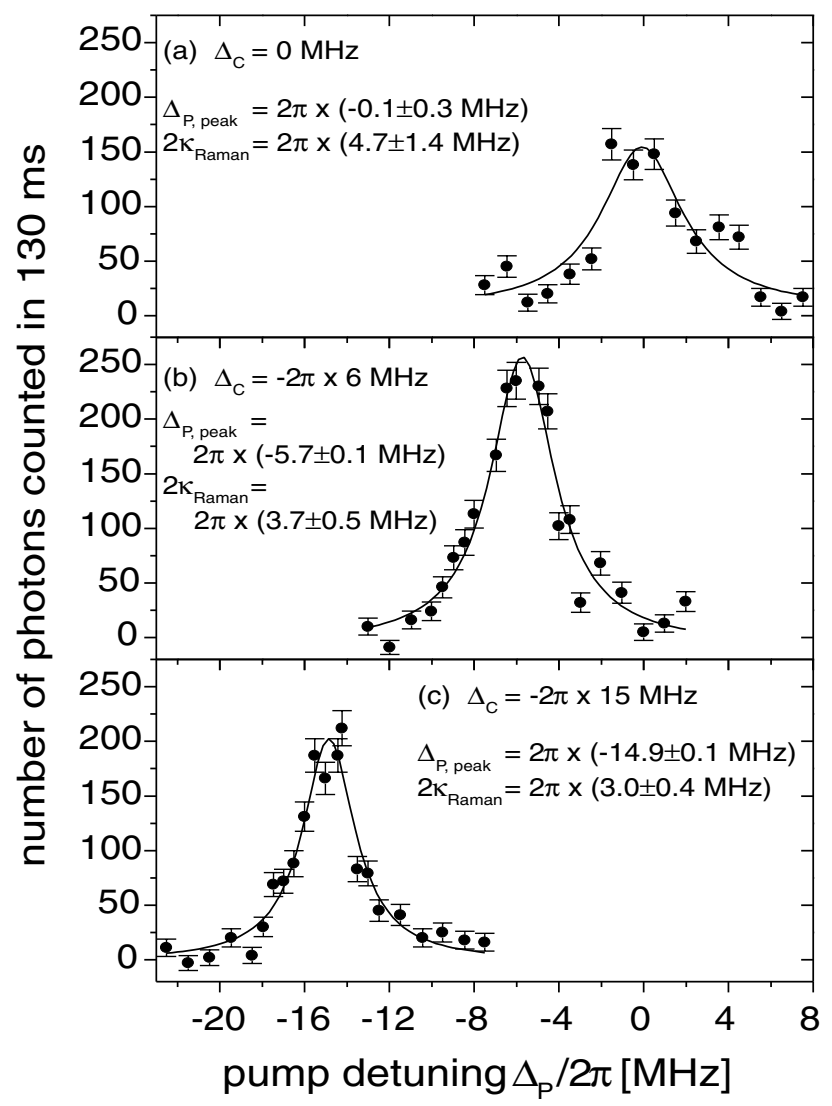

FIG. 5. Number of photons from the cavity as a function of the pump laser detuning, $\Delta_{P}$, for three different cavity detunings. The solid lines are Lorentzian fits to the data. 
the calculated spontaneous emission rate into the cavity is enhanced by a factor of $10^{4}$. However, the linewidth is subnatural, and therefore the observed signal cannot be attributed to an excitation by the pump beam followed by enhanced spontaneous emission.

This is even more evident if the cavity is detuned [Figs. 5(b) and 5(c)]. The emission peak is pulled away from the atomic resonance following the Raman resonance condition, $\Delta_{P}=\Delta_{C}$. Such a displacement proves that the light emission is not the result of a pump transition followed by enhanced spontaneous emission into the cavity. Moreover, $\Delta_{P}$ is too high for an electronic excitation of the atoms. Therefore, the far out-reaching wings of the pump beam no longer excite the atoms prior to their interaction with the cavity mode. The losses vanish, and the peak photon emission probability is higher than for the resonant case. Note also that the observed linewidth is much smaller than the natural linewidth, $\Gamma=2 \pi \times 6 \mathrm{MHz}$, of the atom. For $\Delta_{C}=-2 \pi \times 15 \mathrm{MHz}$, the line is only $3 \mathrm{MHz}$ wide and approaches the linewidth $2 \kappa=2 \pi \times 2.5 \mathrm{MHz}$ of the cavity, which also limits the width of the Raman transition, since $2 \kappa$ is the decay rate of the final state, $|g, 1\rangle$.

In our discussion, we have assumed that the atoms interact with the cavity one-by-one. This is justified according to the following estimation: A mechanical slit restricts the atom's maximum distance from the cavity axis to $\pm 50 \mu \mathrm{m}$. The spatial variation of $g$ along [Fig. 2(d)] and perpendicular to the cavity axis reduces the average emission probability to $37 \%$ per atom crossing the slit and the pump beam. Because of the low quantum efficiency of the SPCM and unavoidable cavity losses, only about $40 \%$ of the generated photons are detected. Therefore the maximum measured rate of 230 events $/ 130 \mathrm{~ms}$ corresponds to a generation rate of 4.4 photons $/ \mathrm{ms}$, and at least 12 atoms/ms are needed to explain this signal. Since the photon generation takes $12 \mu \mathrm{s}$ [FWHM, Fig. 2(b)], the probability that a second atom interacts with the cavity simultaneously is $14 \%$. This is small and, hence, negligible.

All observed features are in excellent agreement with our simulation, and we therefore conclude that the photon emission is caused by a vacuum-stimulated Raman transition, i.e., the coupling to the cavity, $g(t)$, and the Rabi frequency of the pump laser, $\Omega_{P}(t)$, are both high enough to ensure an adiabatic evolution of the system, thus forcing the state vector $|\Psi\rangle$ to follow the dark state $\left|a^{0}\right\rangle$ throughout the interaction. Loss due to spontaneous emission is suppressed, and the photons are emitted into a single mode of the radiation field with well-determined frequency and direction.

The scheme can be used to generate single, wellcharacterized photons on demand, provided the Raman excitation is performed in a controlled, triggered way. In contrast to other single-photon sources [13], these photons will have a narrow bandwidth and a directed emission. Finally, we state that the photon generation process depends on the initial state of the atom interacting with the cavity. If the atom is prepared in a superposition of states $|g, 0\rangle$ and $|u, 0\rangle$, prior to the interaction, this state will be mapped onto the emitted photon. A second atom placed in another cavity could act as a receiver, and, with the suitable pump pulse sequence applied to the emitting and the receiving atom, a quantum teleportation of the atom's internal state could be realized [14].

This work was partially supported by the focused research program, "Quantum Information Processing," of the Deutsche Forschungsgemeinschaft and the QUBITS project of the IST program of the European Union.

[1] A. S. Parkins, P. Marte, P. Zoller, and H. J. Kimble, Phys. Rev. Lett. 71, 3095 (1993); A. S. Parkins, P. Marte, P. Zoller, O. Carnal, and H. J. Kimble, Phys. Rev. A 51, 1578 (1995).

[2] W. Lange and H. J. Kimble, Phys. Rev. A 61, 63817 (2000).

[3] C. K. Law and J.H. Eberly, Phys. Rev. Lett. 76, 1055 (1996); C. K. Law and H. J. Kimble, J. Mod. Opt. 44, 2067 (1997).

[4] A. Kuhn, M. Hennrich, T. Bondo, and G. Rempe, Appl. Phys. B 69, 373 (1999).

[5] For a review, see K. Bergmann and B.W. Shore in Molecular Dynamics and Stimulated Emission Pumping, edited by H.L. Dai and R.W. Field (World Scientific, Singapore, 1995), p. 315-373.

[6] A. Kuhn, S. Steuerwald, and K. Bergmann, Eur. Phys. J. D 1, 57 (1998).

[7] M. Becker, U. Gaubatz, K. Bergmann, and P. L. Jones, J. Chem. Phys. 87, 5064 (1987).

[8] G. Nogues, A. Rauschenbeutel, S. Osnaghi, M. Brune, J. M. Raimond, and S. Haroche, Nature (London) 400, 239 (1999).

[9] B. T. H. Varcoe, S. Brattke, M. Weidinger, and H. Walther, Nature (London) 403, 743 (2000).

[10] H. Mabuchi, Q.A. Turchette, M.S. Chapman, and H. J. Kimble, Opt. Lett. 21, 1393 (1996).

[11] P. Münstermann, T. Fischer, P. W. H. Pinkse, and G. Rempe, Opt. Commun. 159, 63 (1999).

[12] D. J. Heinzen, J. J. Childs, J. E. Thomas, and M. S. Feld, Phys. Rev. Lett. 58, 1320 (1987); D. J. Heinzen and M. S. Feld, Phys. Rev. Lett. 59, 2623 (1987).

[13] J. Kim, O. Benson, H. Kan, and Y. Yamamoto, Nature (London) 397, 500 (1999); C. Brunel, B. Lounis, P. Tamarat, and M. Orrit, Phys. Rev. Lett. 83, 2722 (1999); C. Kurtsiefer, S. Mayer, P. Zarda, and H. Weinfurter, Phys. Rev. Lett. 85, 290 (2000); R. Brouri, A. Beveratos, J.-P. Poizat, and P. Grangier, Opt. Lett. 25, 1294 (2000); P. Michler, A. Imamoğlu, M. D. Mason, P. J. Carson, G.F. Strouse, and S.K. Buratto, Nature (London) 406, 968 (2000).

[14] J. I. Cirac, P. Zoller, H. J. Kimble, and H. Mabuchi, Phys. Rev. Lett. 78, 3221 (1997). 